\title{
Performance Enhancements of Femtosecond Fiber Amplifier by Pump Wavelength Optimization
}

\author{
Sijia Wang $\mathbb{1}$, Peng Qin $\mathbb{1}$, and Huibin Wang $\mathbb{1}$
}

China Academy of Space Technology, Beijing 100094, China

Correspondence should be addressed to Sijia Wang; sj0607@163.com

Received 2 February 2021; Revised 20 February 2021; Accepted 26 February 2021; Published 11 March 2021

Academic Editor: Quan Sheng

Copyright (c) 2021 Sijia Wang et al. This is an open access article distributed under the Creative Commons Attribution License, which permits unrestricted use, distribution, and reproduction in any medium, provided the original work is properly cited.

\begin{abstract}
We demonstrate an efficient scheme to accelerate the self-similar pulse evolution and reduce the intensity noise of a free-running femtosecond fiber amplifier based on the pump wavelength optimization. Experiments and simulations indicate the enhanced tolerances of the pulse self-similar amplification to the seed signal power and pump wavelength fluctuations, with the optimum $915 \mathrm{~nm}$ pump wavelength. $20 \%$ increase in the compressed pulse quality and $\sim 31 \%$ reduction in the amplifier root-mean-square (RMS) relative intensity noise (RIN) $(1.5 \mathrm{kHz}$ to $5 \mathrm{MHz})$ have been observed, even with a more than 4 times higher pump laser diode (LD) RIN than the case of $976 \mathrm{~nm}$. 50 fs transform-limited pulses are generated with the $\sim 0.03 \%$ amplifier RMS RIN. The proposed scheme can lower the requirements of low-noise self-similar femtosecond fiber amplifiers on the power stability of the seed oscillator and the thermal control of the pump LD, thus denoting potentials for the various satellite-based high-precision applications of femtosecond laser in space.
\end{abstract}

\section{Introduction}

High-quality femtosecond laser pulses with high power, short duration, and low noise have important applications in frontier scientific and space technology, such as optical frequency combs, coherent pulse synthesis, high-order harmonic generation, spacecraft networking, and formation flying [1-3]. Ytterbium $(\mathrm{Yb})$ doped fiber self-similar amplifiers feature significant advantages in terms of generating such high-quality femtosecond laser pulses [4-8]. However, the current performances of femtosecond fiber amplifiers are suffering from the finite gain bandwidth at high amplifier gains [9], noise amplifications from the pump laser diode (LD) fluctuations [10], the stimulated Raman scattering (SRS), and amplified spontaneous emission (ASE) disturbances. Lots of advances have been demonstrated in nonlinear femtosecond amplification especially the parabolic self-similar evolution, for high power and short duration. However, there is still lack of efforts to reduce the excessive noise generated in high-gain nonlinear femtosecond fiber amplifiers.

In this paper, a novel self-similar amplification method by optimizing the pump wavelength for high- quality femtosecond laser pulse generation is proposed. The self-similar pulse evolution is efficiently speeded up in a high-gain short fiber amplifier with the reduced intensity noise. Through systematic experiments and numerical simulations under the pump wavelengths of $976 \mathrm{~nm}$ and $915 \mathrm{~nm}$, the parabolic self-similar amplification dynamics and noise suppression mechanisms are explored. The results show that the influence of the seed power noise and the pump wavelength fluctuations of LDs on the pulse amplification and the intensity noise performance can be effectively suppressed due to the wider and flatter absorption cross sections around $915 \mathrm{~nm}$. At the same amplification gain of $20 \mathrm{~dB}$, an optimal pump wavelength of $915 \mathrm{~nm}$ can result in a compressed pulse quality increase of about $20 \%$ and a root-mean-square (RMS) relative intensity noise (RIN) reduction of about $31 \%$ integrated from $1.5 \mathrm{kHz}$ to $5 \mathrm{MHz}$, even though the pump LD RIN is more than 4 times higher. With this approach, transformer limit (TL) pulses of $\sim 50$ fs are generated after compression, and the RMS RIN of the amplifier is $\sim 0.03 \%$. For all we know, the pump wavelength has been optimized to enhance the 
tolerance of pulse self-similar amplification to input signal power and pump fluctuations for the first time. The present study provides a simple method for generating high-quality pedestal-free femtosecond pulses with low noise, thus demonstrating the potential for frontier scientific applications related to high optical coherence and high signal-to-noise ratio. In particular, this scheme can lower the requirements of low-noise self-similar femtosecond fiber amplifiers on the seed power stability from the oscillator and the thermal control for the stabilization of the pump LD. As a result, it would contribute to the various satellite-based high-precision applications of femtosecond laser systems in space.

\section{Experimental Setup and Results}

The schematic diagram of the experimental fiber amplification system is shown in Figure 1. It mainly consists of $1040 \mathrm{~nm}$ ultrashort pulse seed sources, a double-clad (DC) large-mode-area (LMA) fiber amplifier, and a grating-pair compressor. The seed source is made up of a homemade stretched-pulse mode-locked $\mathrm{Yb}$ fiber laser, a diffraction grating pair and a $600-\mathrm{line} / \mathrm{mm}$ diffraction grating pair, and a segment of single-mode fiber (SMF). The output repetition rate of the mode-locked laser oscillator is $74 \mathrm{MHz}$, and the average power is $80 \mathrm{~mW}$. The grating pair works in a double-pass way to widely adjust the chirp and duration of the pulse emitted by the oscillator. Benefitting from this, the interaction between self-phase modulation (SPM) and group velocity dispersion (GVD) in SMF is optimized to shape the pulses temporally and spectrally before the amplifier. At this stage, spectrums of the negative-chirped pulse can be narrowed and smoothed by the interaction with SPM, and temporal durations can also be compressed by the positive GVD [11,12]. The amplifier is composed of an endpumped $2 \mathrm{~m} \mathrm{DC} \mathrm{Yb}$ fiber with a $20 \mu \mathrm{m}$ core. The signal end of the amplifier is spliced to the SMF pigtail in nonlinear pulse preshaper ( $1 \%$ fusion loss). The pump end of the amplifier is spliced to a combiner. The maximum output power of the LDs is $20 \mathrm{~W}$ for both $976 \mathrm{~nm}$ and $915 \mathrm{~nm}$. The compressor is conducted by a Littrow double-pass 600 lines $/ \mathrm{mm}$ grating pair to ensure a $75 \%$ transmission.

A high-resolution spectrometer is employed to monitor and record the pulse spectrums from each stage. With an autocorrelator (AC), the time-domain property of pulses is measured and analyzed. A typically measured feature of self-similar amplified pulses is a high-contrast small-pedestal compressed AC trace generated from a standard grating pair $[13,14]$. The RIN refers to noise of the optical intensity normalized to its average value, and it can be statistically characterized with power spectral density (PSD). The measurement of RIN in this paper is characterized by the use of a standard approach [15]. The RMS values of RIN are calculated for quantitative comparisons. A high-speed photodetector and a $10 \mathrm{MHz}$ low-pass filter are employed to detect the amplified laser signal. To ensure sampling accuracy, the low-frequency $(<100 \mathrm{kHz})$ RIN spectrums are measured by a fast Fourier transform analyzer, and the high-frequency $(>100 \mathrm{kHz})$ spectrums are measured by a radiofrequency spectrum analyzer.

At first, a $976 \mathrm{~nm} \mathrm{LD}$ is used as the pump source in the experiment. The pump power is adjusted for an amplification gain of about $20 \mathrm{~dB}$. The corresponding amplified pulse spectrum and compressed $\mathrm{AC}$ trace are shown by the blue solid curves in Figures 2(a) and 2(b). After that, a 915 $\mathrm{nm} \mathrm{LD}$ is used as the pump source. Experiments are carried out under the same initial conditions, except for the pump power, which is increased to ensure the same $20 \mathrm{~dB}$ gain. The measured amplified pulse spectrums and compressed AC traces are shown by the green solid curves in Figures 2(a) and 2(b). The result of the comparison shows that the appropriate pump wavelength of $915 \mathrm{~nm}$ ensures a smoother amplified spectrum and a smaller pulse base after compression, which indicates a more sufficient self-similar amplification. On this basis, we retrieve the compressed pulses at $976 \mathrm{~nm}$ and $915 \mathrm{~nm}$ pump wavelength by the phase and intensity from correlation and spectrum only (PICASO) algorithm [16] in Figures 2(c) and 2(d), respectively. And the corresponding TL profiles are calculated by the measured spectrums assuming a flat phase. The offset between the pulses obtained by PICASO and the TL profiles is reflective of the chirp linearity. Obviously, the FWHM compressed pulse duration for the case of $915 \mathrm{~nm}$ is slightly wider ( $\sim 52 \mathrm{fs})$, but the deviation from the TL quality is much smaller.

To quantify the deviation from the TL quality and hence the evolution towards self-similar amplification, the Strehl ratio defined as the relative peak power between the compressed pulse to the TL pulse is employed and calculated by $S R=\left(1 / \int_{-\infty}^{\infty}\left|A_{P C}(t)\right|^{2} d t\right) /\left(1 / \int_{-\infty}^{\infty}\left|A_{F T}(t)\right|^{2} d t\right) . A_{P C}(t)$ is the normalized temporal envelope of the PICASO-retrieved compressed pulse. $A_{F T}(t)$ is the TL pulse obtained from the zero-phase Fourier transform of the measured spectrum. The Strehl ratio attains a maximum of 1 for the best TL quality, consistent with the feature of ideal parabolic amplification. According to Figures 2(c) and 2(d), the calculated Strehl ratio is 0.87 and 0.93 for the $976 \mathrm{~nm}$ and $915 \mathrm{~nm}$ pump wavelength, respectively. The incorporation of an optimum $915 \mathrm{~nm}$ pump wavelength leads to the increase of the Strehl ratio and hence the pulse quality by about $20 \%$. We attribute these results mainly to the different absorption levels of the $\mathrm{Yb}$ fiber around these two pump wavelengths. To obtain the same amplification gain, the required input pump power for $915 \mathrm{~nm}$ is much higher than the case for $976 \mathrm{~nm}$, due to the much smaller absorption cross sections. As a consequence, the gain distribution along the amplifier is different. The higher gain level along the former part of the amplifier backward-pumped at $915 \mathrm{~nm}$ can lead to the higher pulse peak power and hence a faster convergence to the selfsimilar regime.

Then, we investigate the noise performances of the femtosecond fiber amplifier under the pump wavelength of $976 \mathrm{~nm}$ and $915 \mathrm{~nm}$. The measured amplifier RIN spectrums are compared in Figure 3. It is obvious that the amplifier RIN level at $976 \mathrm{~nm}$ (blue solid curve) is much higher than $915 \mathrm{~nm}$ (green solid curve) in the high-frequency regime. The bottom of Figure 3 shows the integrated amplifier RMS 


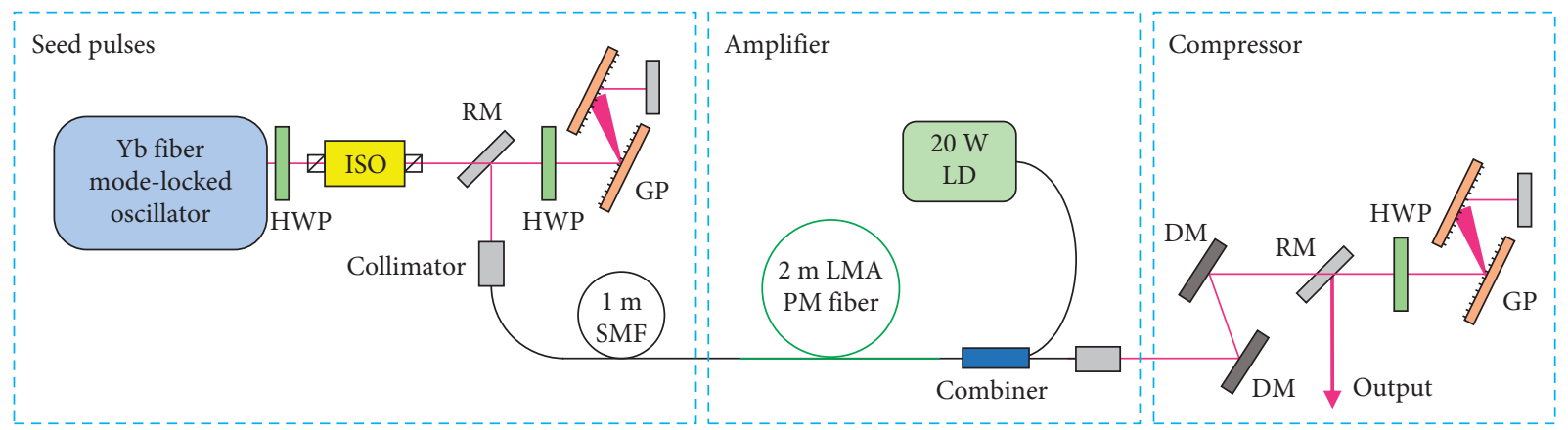

FIGURE 1: Schematic of the experimental setup.

RIN. The value over the frequency range from $1.5 \mathrm{kHz}$ to $5 \mathrm{MHz}$ is $0.042 \%$ and $0.029 \%$ for the $976 \mathrm{~nm}$ and $915 \mathrm{~nm}$ pump wavelength, respectively. Indeed, the incorporation of an optimum $915 \mathrm{~nm}$ pump wavelength results in an approximately $31 \%$ reduction in the RIN value for $976 \mathrm{~nm}$.

To further confirm the benefits of pump wavelength optimization on the amplifier noise suppression, the corresponding RIN of the two pump LDs are measured and compared in the inset of Figure 3. Results show the RMS RIN of the $915 \mathrm{~nm}$ LD (red solid curve) is more than 4 times higher than that of the $976 \mathrm{~nm} \mathrm{LD}$ (gray solid curve). The higher RIN level of $915 \mathrm{~nm}$ pump LD indeed results in the higher RIN level of the amplifier at the low-frequency region $(<1.5 \mathrm{kHz})$, as seen in Figure 3, while for the high-frequency region, the intensity noise of pump LD is supposed to have scarcely any influence on the amplifier RIN performance. The lower amplifier RIN in the condition of pumping at $915 \mathrm{~nm}$ can be attributed partly to the enhanced tolerance of the wavelength fluctuations of pump LD due to the broader and flatter absorption cross section profile around $915 \mathrm{~nm}$. The corresponding lower gain fluctuations along the amplifier can promise smaller noise accumulations from the amplified signal and ASE noise. On the other hand, the higher pulse peak power along the former part of the amplifier pumped by $915 \mathrm{~nm}$ LD can reduce the influence of seed signal power noise on the pulse amplification. Furthermore, the efficient self-similar pulse evolution in this case also attributes to the noise performance due to the inherent stability.

\section{Numerical Simulations}

In the following, numerical simulations are carried out to visualize the pulse evolution inside the amplifier to gain insight into the efficient self-similar pulse evolution and RIN reduction mechanisms. We construct a frequency-resolved numerical model of a backward cladding-pumped nonlinear $\mathrm{Yb}$-doped fiber amplifier. The steady-state two-level rate and power propagation equations including the ASE are employed to describe the wavelength-dependent gain profile. The nonlinear Schrödinger equation (NLSE) is utilized to model the amplified pulse evolution with SPM and GVD. Equations (1)-(6) are the homogeneously broadened propagation equations assuming $\mathrm{CW}$-pumping with no excited state absorption and no background loss:

$$
\begin{aligned}
N_{2}(z) & =\frac{\left\{\left(\lambda_{p} / A_{p} h c\right) \sigma_{a}\left(\lambda_{p}\right) P_{p}(z)+\left(1 / A_{s} h c\right) \sum_{k} \lambda_{k} \sigma_{a}\left(\lambda_{k}\right)\left[P_{s}\left(\lambda_{k}, z\right)+P_{a f}\left(\lambda_{k}, z\right)+P_{a b}\left(\lambda_{k}, z\right)\right]\right\} N_{Y b}}{\left\{\left(\lambda_{p} / A_{p} h c\right)\left[\sigma_{a}\left(\lambda_{p}\right)+\sigma_{e}\left(\lambda_{p}\right)\right] P_{p}(z)+(1 / \tau)+\left(1 / A_{s} h c\right) \sum_{k} \lambda_{k}\left[\sigma_{a}\left(\lambda_{k}\right)+\sigma_{e}\left(\lambda_{k}\right)\right]\left[P_{s}\left(\lambda_{k}, z\right)+P_{a f}\left(\lambda_{k}, z\right)+P_{a b}\left(\lambda_{k}, z\right)\right]\right\}}, \\
N_{1}(z) & =N_{Y b}-N_{2}(z), \\
-\frac{\mathrm{d} P_{p}(z)}{\mathrm{d} z} & =\left[\sigma_{e}\left(\lambda_{p}\right) N_{2}(z)-\sigma_{a}\left(\lambda_{p}\right) N_{1}(z)\right] P_{p}(z) \Gamma_{p} \\
\frac{\mathrm{d} P_{s}(z)}{\mathrm{d} z} & =\sum_{k}\left[\sigma_{e}\left(\lambda_{k}\right) N_{2}(z)-\sigma_{a}\left(\lambda_{k}\right) N_{1}(z)\right] P_{s}\left(z, \lambda_{k}\right) \Gamma_{s}, \\
\frac{\mathrm{d} P_{a f}(z)}{\mathrm{d} z} & =\sum_{k}\left[\sigma_{e}\left(\lambda_{k}\right) N_{2}(z)-\sigma_{a}\left(\lambda_{k}\right) N_{1}(z)\right] P_{a f}\left(\lambda_{k}, z\right) \eta_{s}+\sum_{k} \sigma_{e}\left(\lambda_{k}\right) N_{2}(z) h \frac{c^{2}}{\lambda_{k}^{3}} d \lambda \eta_{s} \\
-\frac{\mathrm{d} P_{a b}(z)}{\mathrm{d} z} & =\sum_{k}\left[\sigma_{e}\left(\lambda_{k}\right) N_{2}(z)-\sigma_{a}\left(\lambda_{k}\right) N_{1}(z)\right] P_{a b}\left(\lambda_{k}, z\right) \eta_{s}+\sum_{k} \sigma_{e}\left(\lambda_{k}\right) N_{2}(z) h \frac{c^{2}}{\lambda_{k}^{3}} \mathrm{~d} \lambda \eta_{s} .
\end{aligned}
$$


Here, $N_{2}(z)$ and $N_{1}(z)$ are the population destinies of the laser ground and upper-level states along the fiber, respectively. $P_{p}(z), P_{s}(z)$, and $P_{a f, b}(z, t)$ represent the average power of the pump, signal, and ASE (forward and backward propagations), respectively. The calculation spectral range is divided into channels, each of which is centered at the wavelength of $\lambda_{k}$. $P_{s}\left(z, \lambda_{k}\right), P_{a f}\left(z, t, \lambda_{k}\right)$, and $P_{a b}\left(z, t, \lambda_{k}\right)$ are the power in the $k$-th channel. With the fiber length $L$ divided into segments, after calculating the power amplification of each spectral channel in the $d z$-segment, the pulse temporal envelope $A(z, t)$ is reobtained from the signal pulse spectral power distribution $P_{s}(\lambda, z)$ through

$$
\begin{aligned}
& A(z, \lambda)=\sqrt{\frac{P_{s}(\lambda, z) \lambda^{2}}{c f d \lambda}} \exp (i \varphi(z, \lambda)), \\
& A(z, t)=\frac{1}{2 \pi} \int_{-\infty}^{\infty} A(z, \omega) \exp (-i \omega t) \mathrm{d} \omega,
\end{aligned}
$$

where $\omega=(2 \pi c / \lambda)$ and $\varphi(z, \lambda)$ is the phase of pulse. As a result, the pulse evolution under SPM and GVD is described by

$$
i \frac{\partial A(z, t)}{\partial z}=\frac{\beta_{2}}{2} \frac{\partial^{2} A(z, t)}{\partial t^{2}}-\gamma|A(z, t)|^{2} A(z, t) .
$$

In this way, the simulations of pulse nonlinear amplification along the gain fiber follow (1) to (9). The fourthorder Runge-Kutta formula is used to solve equations (1) to (6), which are coupled linear differential equations of position $z$. And the split-step Fourier algorithm is the common method to solve the NLSE (9). By the use of an iterative procedure, (1) to (9) can be calculated. The corresponding boundary conditions are set by the initial pump power at the output end of gain fiber and the signal pulse spectral power distribution at the input end. The emission cross sections $\sigma_{e}(\lambda)$ and absorption cross sections $\sigma_{a}(\lambda)$ of the Yb fiber are derived from [17]. Parameters in this model are set according to the experiments. The measured seed pulse in Figure 4 is utilized as the input signal. Figure 4 is the PICASO-reconstructed pulses after the grating prechirper (black solid curve) and the SMF (red solid curve). The chirp (blue dashed curve) after nonlinear preshaping is nearly zero. To quantitatively estimate the efficiency of self-similar parabolic pulse evolution, the misfit parameter $M=\sqrt{\int\left(|A|^{2}-\left|A_{p a}\right|^{2}\right)^{2} \mathrm{~d} t / \int|A|^{4} \mathrm{~d} t}$ is calculated to denote the intensity-profile differences between the pulse $|A|^{2}$ and a parabolic one $\left|A_{p a}\right|^{2}$ with an identical pulse energy and peak power. A smaller $M$ corresponds to a better parabolic fit, and a fully parabolic pulse formation can be determined typically when $M \sim 0.04$.

Simulations under the two pump wavelengths are performed with the input pump power carefully adjusted for the same $20 \mathrm{~dB}$ amplification gain. Figure 4 shows the simulated pulse evolutions of the misfit parameter $M$ along the fiber amplifier, with respect to the pump wavelength of $976 \mathrm{~nm}$ (blue solid curve) and $915 \mathrm{~nm}$ (green solid curve). It is obvious that the $915 \mathrm{~nm}$ pump wavelength indeed leads to a fairly rapid decrease of the misfit parameter $M$ along the fiber amplifier, with a $\sim 30 \%$ reduction of its value for the 976 nm case at the output. Figure 4(d) shows the pulse peak power evolutions, as discussed in the experimental section, pumping at $915 \mathrm{~nm}$ indeed promise the higher pulse peak power along most of the fiber amplifier. Due to the wavelength-dependent absorption cross sections, the gain distribution along the fiber pumped at $976 \mathrm{~nm}$ and $915 \mathrm{~nm}$ is different, even under the same amplification gain. The lower absorption around $915 \mathrm{~nm}$ and the backward-pumped configuration result in higher gains along the former part of the fiber. It in turn leads to a higher pulse peak power, which is beneficial to a faster parabolic self-similar evolution, as confirmed in Figure 4.

At last, a variety of RMS initial relative fluctuations (IRF) are introduced to the signal pulse power and the pump wavelength individually, with respect to the pump wavelength of $976 \mathrm{~nm}$ and $915 \mathrm{~nm}$ in the simulations. The corresponding evolutions of the RMS output relative fluctuations (ORF) of the signal power from the amplifier are calculated and compared in Figure 5. With the RMS IRF of signal power (Figure $5(\mathrm{a})$ ) varied from $0.1 \%$ to $1 \%$, the ORF of signal power increases nearly monotonically for both of the two pump wavelengths. However, the increment of ORF for them is different. Less rapid increase and smaller values of the signal power ORF are found over all signal power IRF for the $915 \mathrm{~nm}$ pump wavelength (green). This enhanced seed signal noise tolerance is a consequence of the higher pulse peak power during amplification and the inherent stability of self-similar pulse evolution, as mentioned previously. While in the condition of the varied pump wavelength IRF (Figure 5(b)), the signal power ORF evolutions for the two pump wavelengths are obviously different. For the $976 \mathrm{~nm}$ pump wavelength (blue), the signal power ORF increases considerably with the increasing pump wavelength IRF, while for the $915 \mathrm{~nm}$ pump wavelength (green), there exhibits a relatively moderate increase of the signal power ORF, due to the much broader and flatter absorption cross section profile than the case of $976 \mathrm{~nm}$. In particular, the signal power ORF induced by the pump wavelength fluctuations around $915 \mathrm{~nm}$ can be more than 30 times smaller than the value for the $976 \mathrm{~nm}$ case. In addition, Figure 5 shows the signal power ORF differences between these two pump conditions increase with the increasing IRF of signal power and pump wavelength. The results above can be seen as the numerical validation of our experimental demonstrations on the faster pulse self-similar evolution, as well as the better amplifier intensity noise performance, enabled by the pump wavelength optimization. In this case, the obviously higher peak power can accelerate the self-similar pulse evolution and reduce the evolution sensitivity to the seed signal power. In the meanwhile, the much broader and flatter absorption cross section profile around $915 \mathrm{~nm}$ can enhance the tolerance of pulse evolution to the fluctuations 


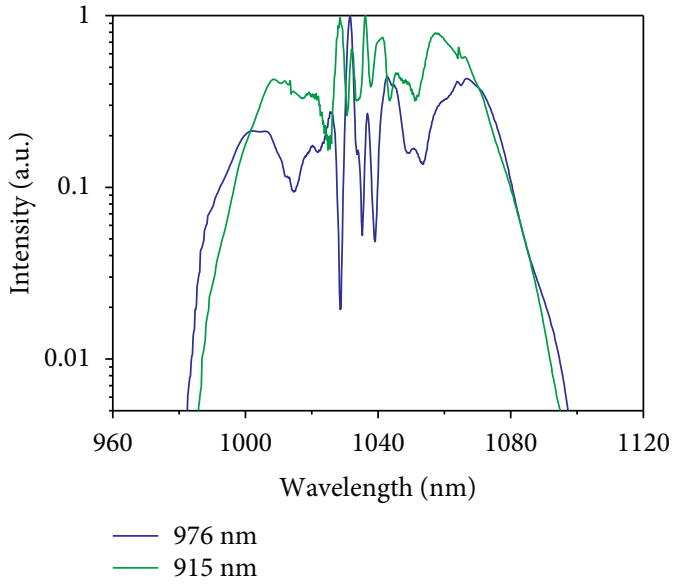

(a)

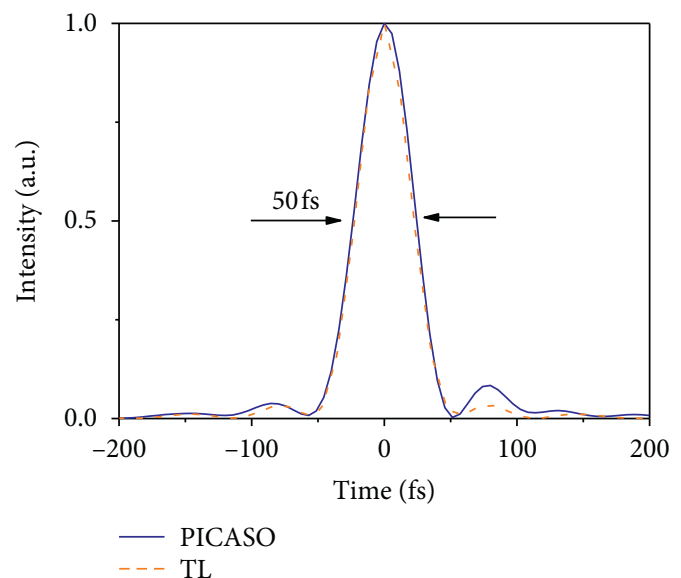

(c)

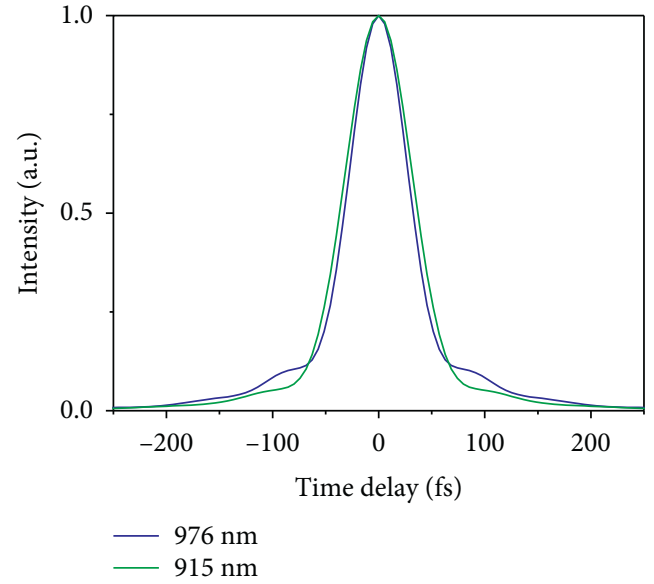

(b)

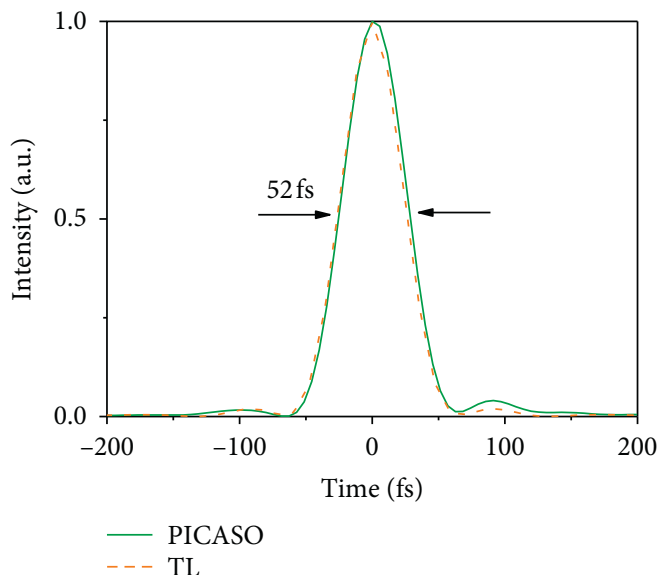

(d)

Figure 2: (a) Measured spectrums and (b) AC traces of the output amplified pulses under a pump wavelength of $976 \mathrm{~nm}$ (blue solid curve) and $915 \mathrm{~nm}$ (green solid curve). PICASO-retrieved (solid curves) and transform-limited (dashed curves) pulse under a pump wavelength of (c) $976 \mathrm{~nm}$ and (d) $915 \mathrm{~nm}$.

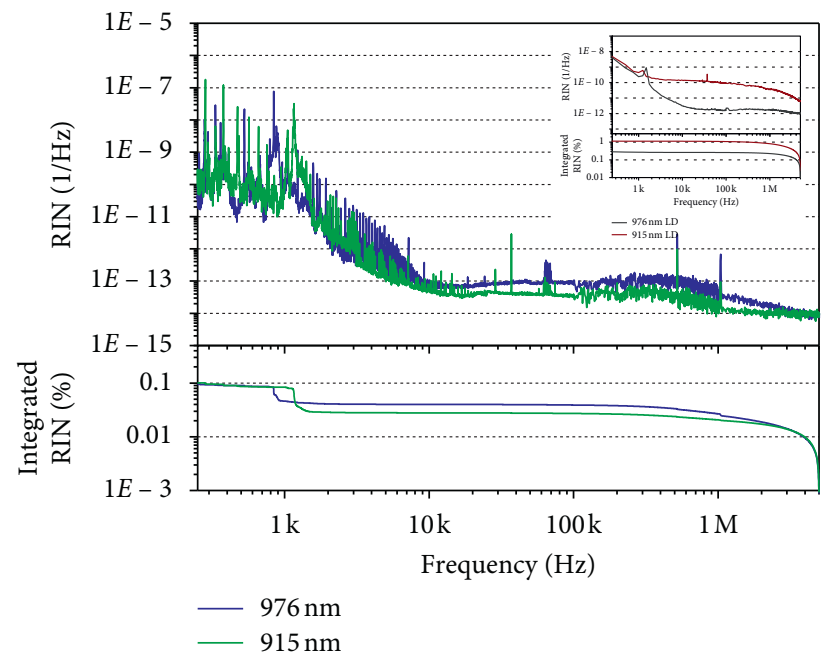

Figure 3: Amplifier RIN spectrums measured at pump wavelengths of $976 \mathrm{~nm}$ (blue solid curves) and $915 \mathrm{~nm}$ (green solid curves). Inset: measured RIN spectrums of the $976 \mathrm{~nm} \mathrm{LD} \mathrm{(gray} \mathrm{solid} \mathrm{curve)} \mathrm{and} \mathrm{the} 915 \mathrm{~nm}$ LD (red solid curve). 

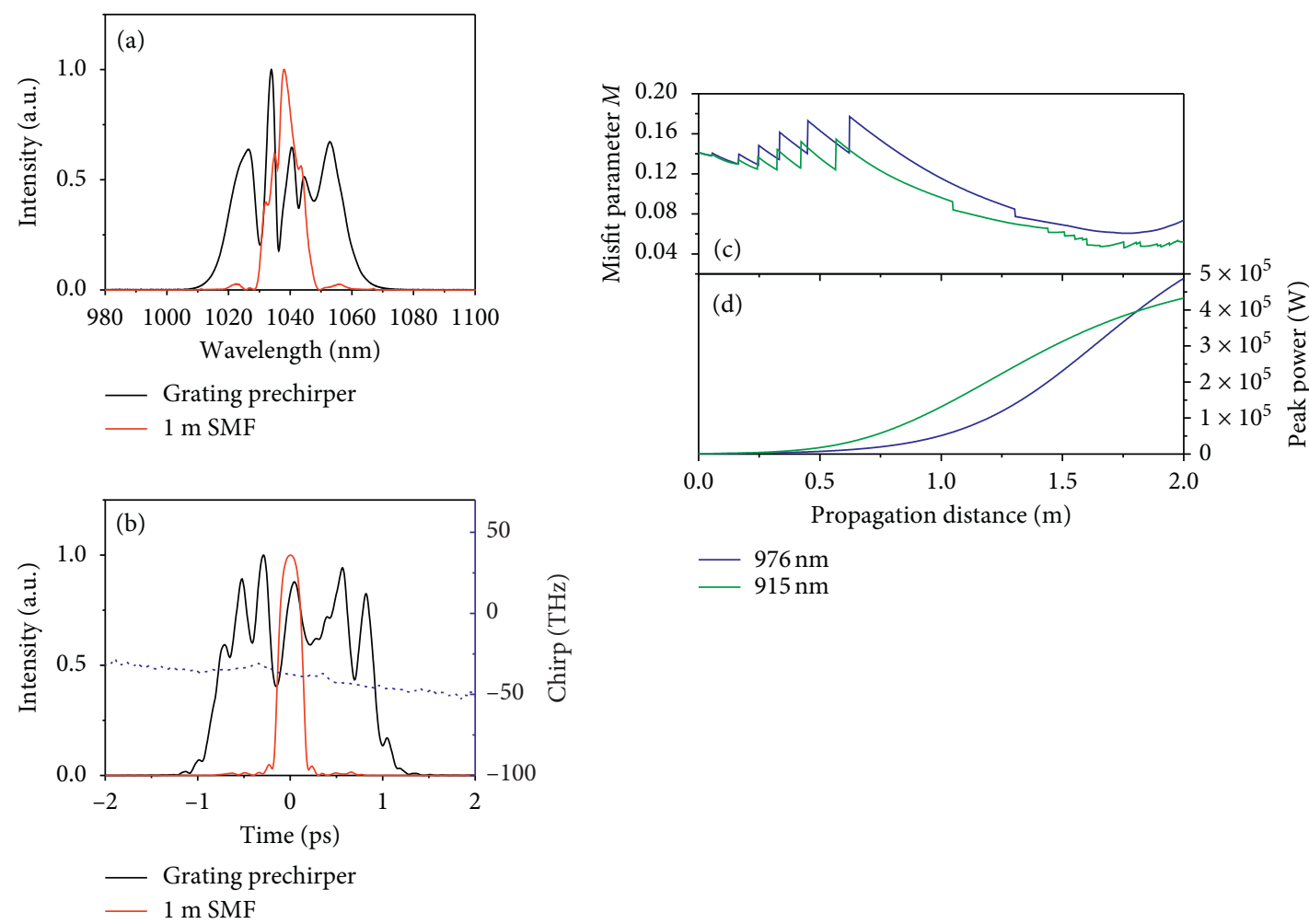

Figure 4: Simulations with (a) the measured spectrums and (b) the PICASO-reconstructed pulses output from the grating prechirper (black solid curve) and the SMF (red solid curve) as the input signal. Amplified pulse evolutions along the fiber under a pump wavelength of $976 \mathrm{~nm}$ (blue solid curve) and $915 \mathrm{~nm}$ (green solid curve): (c) the misfit parameter and (d) the pulse peak power.

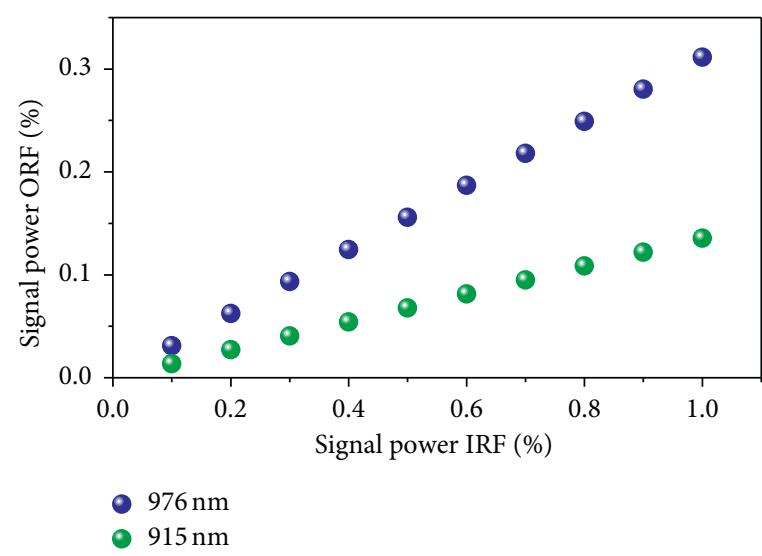

(a)

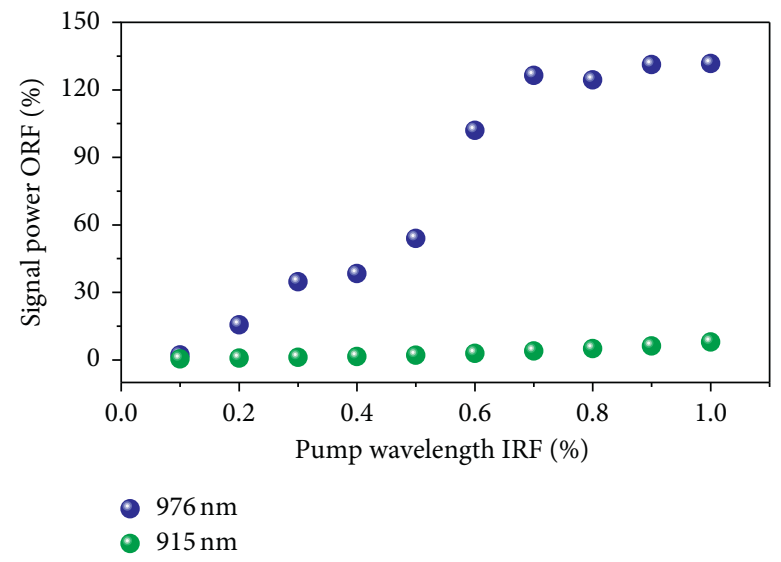

(b)

FIGURE 5: Simulated ORF of the signal power from the fiber amplifier versus the IRF of (a) signal power and (b) pump wavelength, with respect to the pump wavelength of $976 \mathrm{~nm}$ (blue) and $915 \mathrm{~nm}$ (green).

of pump wavelength. Therefore, benefiting from an optimum $915 \mathrm{~nm}$ pump wavelength, the efficient self-similar pulse evolution and reduction of the influences of the seed signal power and the pump wavelength fluctuations have evidently led to a low-noise operation.

\section{Conclusions}

We have demonstrated a simple passive scheme to generate femtosecond pulses with both the ultrashort pedestal-free duration and low-intensity noise from short-length fiber 
amplifiers. It is based on the efficient self-similar pulse evolution and amplifier RIN reduction enabled by the pump wavelength optimization. Results show that under the same $20 \mathrm{~dB}$ amplification gain, the optimum $915 \mathrm{~nm}$ pump wavelength can not only accelerate the self-similar evolution but also enhance the tolerance of fluctuations of the seed signal power and pump wavelength, leading to a $20 \%$ increase in the compressed pulse quality and $\sim 31 \%$ reduction in the RMS RIN (1.5 kHz to $5 \mathrm{MHz})$. $~ 50$ fs transformlimited pulses are generated with the $\sim 0.03 \%$ RMS RIN. To the best of our knowledge, the pump wavelength has been optimized experimentally and numerically for the first time to enhance the tolerances of pulse self-similar amplification to the seed signal power and pump wavelength fluctuations. In particular, the proposed scheme can lower the requirements of low-noise self-similar femtosecond fiber amplifiers on the seed pulse power stability from the oscillator and the thermal control of the pump LD, thus denoting potentials for the various space-based high-precision applications of femtosecond laser.

\section{Data Availability}

The data used to support the findings of this study are included within the article.

\section{Disclosure}

Part of the research described in this manuscript was presented at a conference in 2017, Conference on Lasers and Electro-Optics Pacific Rim (CLEO-PR).

\section{Conflicts of Interest}

The authors declare that there are no conflicts of interest regarding the publication of this paper.

\section{Acknowledgments}

This work was funded by the National Science and Technology Innovation Special Zone Program of China, Independent Innovation Project of China Academy of Space Technology, Science and Technology Innovation Research and Development Project of China Aerospace Science and Technology Corporation.

\section{References}

[1] J. Kim and Y. Song, "Ultralow-noise mode-locked fiber lasers and frequency combs: principles, status, and applications," Advances in Optics and Photonics, vol. 8, no. 3, pp. 465-540, 2016.

[2] H. Carstens, M. Högner, T. Saule et al., "High-harmonic generation at $250 \mathrm{MHz}$ with photon energies exceeding $100 \mathrm{eV}$," Optica, vol. 3, no. 4, pp. 366-369, 2016.

[3] M. Guggenmos, K. Safak, M. Y. Peng et al., "Attosecond precision multi-kilometer laser-microwave network," Light: Science \& Applications, vol. 6, Article ID e16187, 2017.

[4] H. Song, B. Liu, W. Chen et al., "Femtosecond laser pulse generation with self-similar amplification of picosecond laser pulses," Optics Express, vol. 26, no. 20, pp. 26411-26421, 2018.
[5] H. Li, Z. Cheng, R. Sun et al., "172-fs, 27- JJ, Yb-doped allfiber-integrated chirped pulse amplification system based on parabolic evolution by passive spectral amplitude shaping," Optics Express, vol. 27, no. 23, pp. 34103-34112, 2019.

[6] M. E. Peng, V. I. Kruglov, B. C. Thomsen, J. M. Dudley, and J. D. Harvey, "Self-similar propagation and amplification of parabolic pulses in optical fibers," Physical Review Letters, vol. 84, no. 26, pp. 6010-6013, 2000.

[7] A. J. Verhoef, K. Jespersen, T. V. Andersen et al., "High peakpower monolithic femtosecond ytterbium fiber chirped pulse amplifier with a spliced-on hollow core fiber compressor," Optics Express, vol. 22, no. 14, pp. 16759-16766, 2014.

[8] S. Grüner-Nielsen, B. Liu, C. Gu et al., "Self-similar evolution in a short fiber amplifier through nonlinear pulse preshaping," Optics Letters, vol. 38, no. 3, pp. 296-298, 2013.

[9] S. Wang, W. Chen, P. Qin, Y. Song, M. Hu, and B. Liu, "Spectral and temporal breathing self-similar evolution in a fiber amplifier for low-noise transform-limited pulse generation," Optics Letters, vol. 41, no. 22, pp. 5286-5289, 2016.

[10] P. Song, S. Wang, M. Hu, and Y. Song, "Passive optimization of pump noise transfer function by narrow band-pass filtering in femtosecond fiber lasers," High Power Laser Science and Engineering, vol. 7, p. e52, 2019.

[11] E. R. Andresen, J. M. Dudley, D. Oron, C. Finot, and H. Rigneault, "Transform-limited spectral compression by self-phase modulation of amplitude-shaped pulses with negative chirp," Optics Letters, vol. 36, no. 5, pp. 707-709, 2011.

[12] D. A. Korobko, O. G. Okhotnikov, and I. O. Zolotovskii, "Multistage fiber preamplifier employing spectral compression for generation of high-energy laser pulses," Journal of the Optical Society of America B, vol. 33, no. 2, pp. 239-245, 2016.

[13] S. Wang, B. Liu, M. Hu, and C. Wang, "On the efficiency of parabolic self-similar pulse evolution in fiber amplifiers with gain shaping," Journal of Lightwave Technology, vol. 34, no. 13, pp. 3023-3034, 2016.

[14] C. Finot, F. Parmigiani, P. Petropoulos, and D. Richardson, "Parabolic pulse evolution in normally dispersive fiber amplifiers preceding the similariton formation regime," Optics Express, vol. 14, no. 8, pp. 3161-3170, 2006.

[15] P. Qin, Y. Song, H. Kim et al., "Reduction of timing jitter and intensity noise in normal-dispersion passively mode-locked fiber lasers by narrow band-pass filtering," Optics Express, vol. 22, no. 23, pp. 28276-28283, 2014.

[16] J. W. Shin and W. Rudolph, "Noise sensitivity and accuracy of femtosecond pulse retrieval by phase and intensity from correlation and spectrum only (PICASO)," Journal of the Optical Society of America B, vol. 19, no. 2, pp. 330-339, 2002.

[17] R. Paschotta, J. Nilsson, A. C. Tropper, and D. C. Hanna, "Ytterbium-doped fiber amplifiers," IEEE Journal of Quantum Electronics, vol. 33, no. 7, pp. 1049-1056, 1997. 